\title{
Valor preditor de morbimortalidade da relação neutrófilo-linfócito em pacientes com doença renal crônica: revisão integrativa da literatura
}

\author{
Morbidity and mortality predictive value of the neutrophil-lymphocyte ratio in patients with \\ chronic kidney disease: an integrative literature review \\ Valor predictivo de morbilidad y mortalidad del ratio neutrófilos-linfocitos en pacientes con \\ enfermedad renal crónica: una revisión bibliográfica integradora
}

Igor Henrique Silva Soares ORCID: https://orcid.org/0000-0001-6784-4660

Universidade de Uberaba, Brasil

E-mail: igorhenrique506@gmail.com

Danielle Silva Borges

ORCID: https://orcid.org/0000-0002-6766-5918

Universidade de Uberaba, Brasil

E-mail: silvabborgesdanielle@gmail.com

Gabriela Silva Moreira

ORCID: https://orcid.org/0000-0002-9240-6352 Universidade de Uberaba, Brasil

E-mail: g-silva-moreira@hotmail.com

Giovanna Ferreira Gomes

ORCID: https://orcid.org/0000-0001-6511-4664 Universidade de Uberaba, Brasil

E-mail: giovannafgomes2711@ hotmail.com

Luísa Lyrio Stábille

ORCID: https://orcid.org/0000-0001-7061-5455 Universidade de Uberaba, Brasil

E-mail: luisa_lyrio@ hotmail.com

Eduardo Almeida Pedrosa

ORCID: https://orcid.org/0000-0003-1226-9640 Universidade Federal do Triângulo Mineiro, Brasil

E-mail: eduardoalmeidapedrosa@gmail.com

Rafael Freitas Silva Peralta

ORCID: https://orcid.org/0000-0001-6170-830X Centro Universitário de Patos de Minas, Brasil E-mail: peraltarfs@gmail.com

Douglas Reis Abdalla

ORCID: https://orcid.org/0000-0002-6971-1201 Universidade de Uberaba, Brasil Faculdade de Talentos Humanos, Brasil E-mail: drabdalla@facthus.edu.br

\begin{abstract}
Resumo
A Doença Renal Crônica (DRC) e suas complicações causam um grande impacto na qualidade de vida dos pacientes acometidos, podendo ocasionar óbito. As relação de neutrófilos-linfócitos (NLR), proporção de linfócitos-monócitos (LMR), relação plaquetas-linfócitos (PLR) e volume plaquetário médio (MPV) podem ser usados como fatores determinantes do prognóstico de pacientes em várias situações clínicas. O objetivo desta revisão foi buscar na literatura evidências acerca das relações celulares (NLR, LMR, PLR e MPV) e sua correlação com a DRC. Para tanto, foi consuzida uma revisão integrativa da literatura nas bases de dados LILACS, MEDLINE, PubMed e CINAHL das produções dos últimos 10 anos. Os descritores utilizados foram: "doença renal crônica", "lesão renal crônica", "insuficiência renal crônica" "proporção monócitos para linfócitos", "proporção monócitos-linfócitos”, "proporção neutrófilos para linfócitos" e "proporção neutrófilos-linfócitos", os quais foram conjugados para delimitação da busca. Foram selecionados 26 artigos ao final das etapas de busca. Sendo o ano de 2019 o mais prevalente com 19,23\% das publicações, seguido pelo ano de 2013 e 2016 com 15,38\% das publicações. Em relação ao país de origem dos estudos, 53,8\% foram conduzidos na Turquia, seguidos pelos estudos realizados na China com 15,38\% e Estados Unidos, Taiwan e Japão com 7,69\% em cada país . A maioria dos estudos apresentaram dados que demonstram que as relações celulares como LMR, PLR, MPV e principalmente NRL são instrumentos práticos e podem ser utilizadas como marcadores de inflamação sistêmica e preditores de morbimortalidade na DRC, sobretudo aqueles pacientes em
\end{abstract}


com a doença em estágio final. Portanto, foi evidenciado que há um aumento das relações celulares nos pacientes com DRC. Além disso, esse é um método com ótimo custo benefício para predizer inflamação e mortalidade. Porém novos estudos são necessários para ampliar seu uso na prática clínica.

Palavras-chave: Doença renal crônica; Relações celulares; Inflamação.

\begin{abstract}
Chronic kidney disease (CKD) and its complications have a major impact on the quality of life of affected patients, and may lead to death. The neutrophil-lymphocyte ratio (NLR), lymphocyte-monocyte ratio (LMR), plateletlymphocyte ratio (PLR) and mean platelet volume (MPV) can be used as prognostic determinants of patients in various clinical situations. The aim of this review was to search the literature for evidence about cellular ratios (NLR, LMR, PLR and MPV) and their correlation with CKD. To this end, an integrative literature review was carried out in LILACS, MEDLINE, PubMed, and CINAHL databases of productions from the last 10 years. The descriptors used were: "chronic kidney disease", "chronic kidney injury", "chronic renal failure", "monocyte to lymphocyte ratio", "monocyte to lymphocyte ratio", "neutrophil to lymphocyte ratio", and "neutrophil to lymphocyte ratio", which were combined to delimit the search. Twenty-six articles were selected at the end of the search steps. Being the year 2019 the most prevalent with $19.23 \%$ of publications, followed by the year 2013 and 2016 with $15.38 \%$ of publications. Regarding the country of origin of the studies, $53.8 \%$ were conducted in Turkey, followed by studies conducted in China with $15.38 \%$ and the United States, Taiwan and Japan with $7.69 \%$ in each country . Most studies presented data showing that cellular ratios such as MRL, PLR, MPV and especially NRL are practical tools and can be used as markers of systemic inflammation and predictors of morbidity and mortality in CKD, especially those patients with end-stage disease. Therefore, it was evidenced that there is an increase in cellular ratios in CKD patients. Moreover, this is a cost-effective method to predict inflammation and mortality. However, further studies are needed to expand its use in clinical practice.
\end{abstract}

Keywords: Chronic kidney disease; Cellular relationships; Inflammation.

\begin{abstract}
Resumen
La Enfermedad Renal Crónica (ERC) y sus complicaciones causan un gran impacto en la calidad de vida de los pacientes afectados, y pueden causar la muerte. El cociente neutrófilos-linfocitos (NLR), el cociente linfocitosmonocitos (LMR), el cociente plaquetas-linfocitos (PLR) y el volumen medio de plaquetas (MPV) pueden utilizarse como determinantes del pronóstico de los pacientes en diversas situaciones clínicas. El objetivo de esta revisión fue buscar en la literatura evidencia sobre los ratios celulares (NLR, LMR, PLR y MPV) y su correlación con la ERC. Para ello, se realizó una revisión bibliográfica integradora en las bases de datos LILACS, MEDLINE, PubMed y CINAHL de producciones de los últimos 10 años. Los descriptores utilizados fueron: "doença renal crônica", "lesão renal crônica", "insuficiência renal crônica", "proporção monócitos para linfócitos", "proporção monócitos-linfócitos", "proporção neutrófilos para linfócitos" y "proporção neutrófilos-linfócitos", los cuales fueron conjugados para delimitar la búsqueda. Al final de las etapas de búsqueda se seleccionaron 26 artículos. Siendo el año 2019 el más prevalente con el 19,23\% de las publicaciones, seguido del año 2013 y 2016 con el 15,38\% de las publicaciones. En cuanto al país de origen de los estudios, el 53,8\% se realizó en Turquía, seguido de los estudios realizados en China, con un $15,38 \%$, y Estados Unidos, Taiwán y Japón, con un 7,69\% en cada país. La mayoría de los estudios presentaron datos que mostraban que los ratios celulares, como el LMR, el PLR, el MPV y, especialmente, el NRL, son herramientas prácticas y pueden utilizarse como marcadores de inflamación sistémica y predictores de morbilidad y mortalidad en la ERC, especialmente en aquellos pacientes con enfermedad en fase terminal. Por lo tanto, se evidenció que existe un aumento de los ratios celulares en los pacientes con ERC. Además, se trata de un método rentable para predecir la inflamación y la mortalidad. Sin embargo, se necesitan más estudios para ampliar su uso en la práctica clínica.
\end{abstract}

Palabras clave: Enfermedad renal crónica; Relaciones celulares; Inflamación.

\title{
1. Introdução
}

A doença renal crônica (DRC) constitui um problema importante no Brasil, por ter aumento progressivo nas taxas de prevalência e incidência. Os rins são órgãos essências na manutenção da homeostase do corpo humano e, com a diminuição da taxa de filtração glomerular, além de ocorrer prejuízo das funções regulatórias, excretórias e endócrinas, tem-se o comprometimento também de todos os outros órgãos do organismo (Porto et. al, 2017). Portanto, independente da etiologia, essa doença causa várias complicações para os pacientes o que afeta a qualidade de vida, podendo causar óbitos em casos graves.

De acordo com Aguiar et al. (2020), a DRC é considerada um grande agravo para a saúde pública, sendo uma das principais comorbidades responsáveis pela mudança do perfil epidemiológico no Brasil. Diante disso, foi realizada a Pesquisa 
Nacional de Saúde (PNS), em que a prevalência de DRC autorreferida é de 1,42\%, ou seja, em torno de dois milhões de brasileiros possuem a lesão renal, comprovando assim, a magnitude da doença. Além disso, há uma maior prevalência da DRC em pacientes tabagistas e sedentários, hipertensos (2,85\%), diabéticos $(3,49 \%)$ e dislipidemicos $(3,60 \%)$. Outro ponto relevante, é a doença ser mais prevalente no sexo feminino (1,48\%) e em indivíduos com faixa etária acima de 65 anos $(3,13 \%)$.

As principais causas para desenvolvimento da DRC são diabetes, hipertensão, glomerulonefrite crônica, pielonefrite crônica, uso crônico de anti-inflamatórios não esteroides, doenças autoimunes, doença renal policística, síndrome de Alport, malformações congênitas e lesão renal aguda prolongada (Ammirati, 2020). Além disso, a albuminúria e o prejuízo da função renal são preditores de DRC para pacientes com doenças cardiovasculares. Segundo Aguiar et al. (2020), acredita-se que no Brasil a DRC também está associada a condições sociodemográficas, estilo de vida não saudável e doenças crônicas. O controle da pressão arterial, da glicemia e proteinúria são fatores relacionados à prevenção da progressão da doença.

Visto que a DRC está relacionada à perda permanente e progressiva da função renal, essa patologia, segundo Morsch e Veronese (2011), leva a um quadro clínico de sinais e sintomas característico no qual o paciente apresenta anemia relacionada a menor produção renal de eritropoietina, alterações no balanço hidroeletrolítico corporal que levam ao aumento da pressão arterial e da retenção de algumas moléculas (entre elas ureia, creatinina e potássio) e, quando há presença de lesão renal, pode-se ter um quadro de proteinúria. Outros sinais comuns são o enfraquecimento ósseo em decorrência da menor ativação renal de vitamina D e da hipertrofia das paratireoides e, por fim, alterações neurológicas (náuseas, cefaléia, distúrbios do sono) em virtude do acúmulo de moléculas tóxicas.

Segundo Kirsztajn et al. (2011), a DRC é definida a partir da avaliação funcional e estrutural dos rins. O diagnóstico é baseado em dois critérios: (1) Anormalidades estruturais ou funcionais que evidenciam lesão, há três meses ou mais, acompanhada ou não da diminuição da taxa de filtração glomerular (TFG). Nesse caso, a lesão é avaliada através de anormalidades histopatológicas ou de marcadores de lesão renal (alterações sanguíneas, urinárias ou imaginológicas). (2) Diminuição da TFG abaixo de $60 \mathrm{~mL} / \mathrm{min} / 1,73 \mathrm{~m} 2$ há três meses ou mais, acompanhada ou não de lesão renal.

Segundo Bastos; Bregman e Kirsztajn (2010), no Brasil, a incidência e a prevalência de DRC estão aumentando, o prognóstico ainda é ruim e os custos do tratamento da doença são altíssimos. Independentemente da doença de base, os principais desfechos em pacientes com DRC são as suas complicações (anemia, acidose metabólica, alteração do metabolismo mineral e desnutrição), decorrentes da perda funcional renal, óbito (principalmente por causas cardiovasculares) e falência funcional dos rins. Estudos recentes indicam que estes desfechos indesejados podem ser prevenidos ou retardados se a DRC for diagnosticada precocemente e as medidas nefro e cardioprotetoras implementadas precocemente. Porém, a DRC é subdiagnosticada e tratada inadequadamente, resultando na perda de oportunidade para a implementação de prevenção primária, secundária e terciária, em parte devido à falta de conhecimento da definição e classificação dos estágios da doença, bem como a não utilização de testes simples para o diagnóstico e avaliação funcional da doença.

Segundo Lee et al. (2018), a partir da correlação entre estado inflamatório e doença ou prognóstico do câncer, há um interesse crescente em pesquisas voltadas para compreender melhor o estado da doença ou prever o prognóstico de pacientes com exames de sangue simples. Dessa forma, A proporção de neutrófilos-linfócitos (NLR), proporção de linfócitos-monócitos (LMR), relação plaquetas-linfócitos (PLR) e volume plaquetário médio (MPV) podem ser usados como fatores para determinar o prognóstico de pacientes em várias situações clínicas. Como um marcador de inflamação sistêmica, NLR demonstrou ser eficaz na previsão do prognóstico de tratamentos de câncer, intervenções coronárias, cirurgia de revascularização do miocárdio e Doença de Alzheimer. Já o LMR, PLR E MPV demonstraram prognóstico em pacientes graves no pós-operatório e tratamento intensivo. A partir disso, existe a possibilidade de identificar uma doença ou prever o status de saúde usando essas relações celulares, porém ainda existem muitas variáveis que impedem uma padronização de valores comparados a pacientes 
saudáveis.

Ainda não há um consenso sobre qual marcador está mais relacionado à inflamação na DRC. Atualmente, o mais utilizado é a proteína C reativa (PCR), apesar de que novos estudos têm trazido a NLR como um novo preditor de inflamação nesta patologia, além de demonstrarem que a relação tem valores prognósticos e prenunciados de morbimortalidade, principalmente em indivíduos com inflamação sistêmica (Okyay et. al, 2013). A pesquisa, realizada por Altunoren et. al (2018), evidenciou que pacientes com NLR mais elevado, apresentaram maior redução anual de taxa de filtração glomerular (TGF), menores valores de albumina, elevação de PCR e proteinúria aumentada. Além disso, a sobrevida renal dos pacientes com a NRL mais elevada, foi menor do que em indivíduos com valores baixos da mesma. A elevação desta razão ainda relaciona-se à maior inflamação e desnutrição, e piora do perfil clínico, o que contribui para um estágio avançado da doença renal.

O MPV é outro marcador relacionado à inflamação na DRC. Apesar de não haver comprovação de uma diferença relevante entre valores de MPV para doentes renais crônicos e pessoas saudáveis, valores elevados de MPV indicaram menores taxas de TGF (Ylmaz et.al, 2017).

Desta forma, o presente artigo visa buscar na literatura evidências acerca das relações celulares (NLR, LMR, PLR e MPV) e sua correlação com a DRC.

\section{Metodologia}

No presente estudo foi conduzida uma revisão integrativa, que consiste em uma pesquisa que permite a partir de evidências a avaliação, síntese e conhecimento acerca de um fenômeno, objetivando produzir uma visão geral de conceitos complexos, teorias ou problemas de saúde relevantes a partir de estudos pré-existentes, possibilitando a proposição de intervenção (Galvão et al., 2004; Whittemore; Knafl, 2005).

Para a seleção dos artigos, foram conduzidas 6 etapas metodológicas, quais sejam: 1. elaboração da questão norteadora ou hipótese da pesquisa, ou seja, identificou-se o problema, apresentou-se o mecanismo de busca e os descritores ou palavras chave; 2. estabelecimento dos critérios de inclusão e exclusão dos artigos a serem selecionados para composição da amostra; 3. leitura exploratória dos títulos e resumos dos artigos para pré-seleção; 4. leitura analítica dos artigos a fim de compilar, analisar e categorizar as informações; 5. interpretação dos resultados. 6. síntese seguida da apresentação dos resultados identificados, que permeiam a questão norteadora (De Sousa et al., 2011).

Portanto, neste estudo optou-se por realizar busca sobre os conceitos: chronic kidney disease, chronic kidney injury, chronic kidney failure monocyte to lymphocyte ratio, monocyte-lymphocyte ratio, neutrophil to lymphocyte ratio, neutrophillymphocyte ratio. A partir desses conceitos, definiu-se a questão norteadora: Quais os achados na literatura sobre a conformidade existente entre as relações RNL, RML, RPL e seus significados na Doença Renal Crônica?

Após a formulação da questão a ser pesquisada, foi realizado um levantamento bibliográfico na plataforma PubMed. O levantamento do estudo ocorreu entre abril e maio de 2021. E a seleção dos textos procedeu com as buscas na plataforma, utilizando os filtros nela disponíveis para textos publicados entre 2010 e 2021. Para seleção das publicações, foram adotados os seguintes critérios de inclusão: artigos científicos, publicados no idioma Inglês, entre os anos de 2010 a 2020 , disponíveis online e gratuitamente na íntegra. Foram excluídos os artigos sem resumo na base de dados ou incompletos, editoriais, cartas ao editor, estudos reflexivos, revisões sistemáticas ou integrativas de literatura.

Após a definição da questão norteadora, localização e seleção dos artigos, foram identificadas 10918 publicações potencialmente elegíveis para serem incluídas nessa revisão. Após a aplicação dos critérios de inclusão e exclusão a amostra foi composta por 528 publicações, foram analisados os resumos de 30 registros, para verificar se atenderiam os critérios de elegibilidade e se responderiam à pergunta que norteia esta revisão, assim excluiu-se 4 registros e somente 26 foram analisados 
na íntegra para confirmar a elegibilidade para a síntese quantitativa e análise dos dados conforme o fluxograma de seleção (Figura 1).

Figura 1: Fluxograma de Seleção das Publicações.

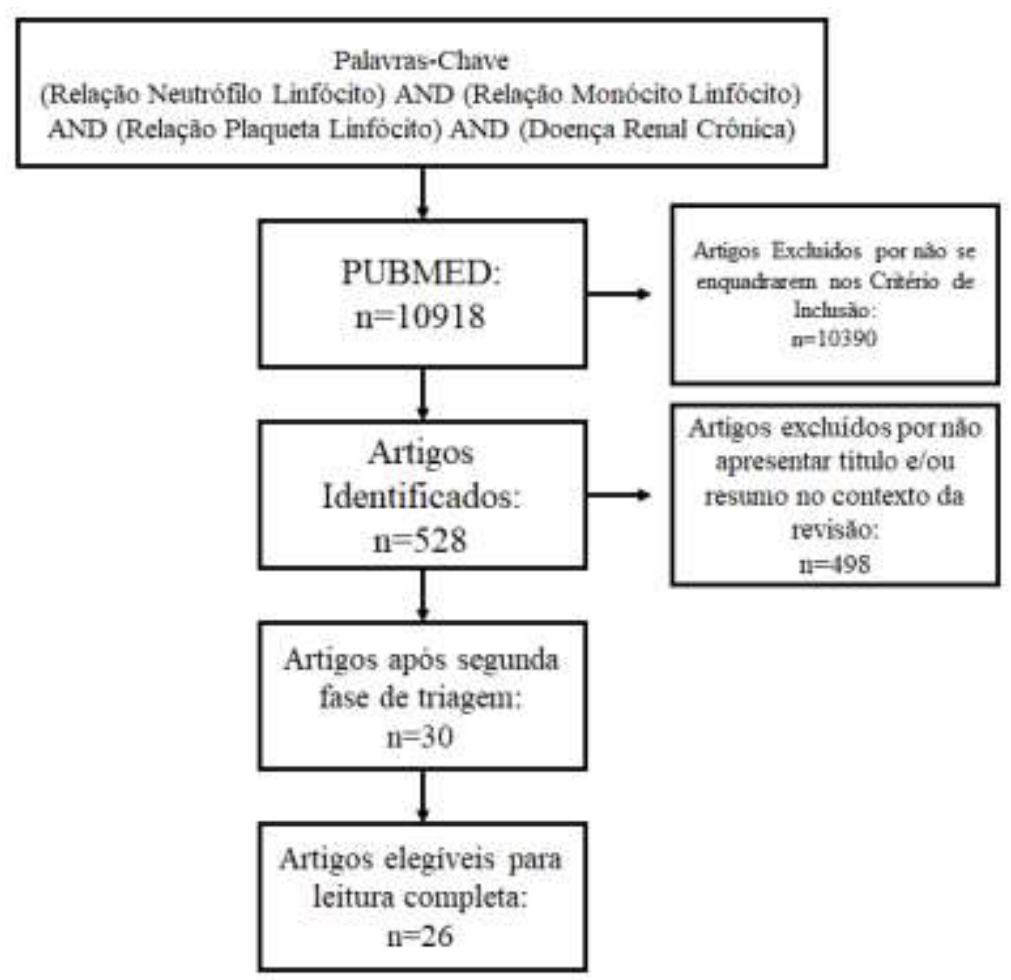

Fonte: Autores (2021).

\section{Resultados e Discussão}

No espaço de tempo delimitado para a realização deste estudo (2010-2020) foram encontradas e analisadas 26 publicações. Em 2 sumarizados no Quadro 1, 2012 e 2015 foram publicados 1 artigo (3,84\%) em cada ano respectivamente. Nos anos de 2017, 2018 e 2020 consta 3 publicações em cada ano (11,53\%). Já nos anos de 2013, 2014,2016 e 2019 foram publicados $4(15,38 \%), 2(7,69 \%), 4(15,38 \%)$ e $5(19,23 \%)$ artigos respectivamente.

De acordo com a metodologia dos trabalhos selecionados os tipos de estudo eram pesquisas clínicas utilizando pacientes saudáveis comparando com indivíduos com doença renal crônica.

As publicações resultaram de diferentes revistas sendo: Folia Med Cracov; J Transl Med; Arch Med Sci; Clin Chim Acta; Ren Fail; Nefrologia; Clin Exp Nephrol; Saudi J Kidney Dis Transpl; Acta Cardiol Sin; Acta Clin Belg; Int Immunopharmacol; Int Urol Nephrol; Kidney Blood Press Res; J Clin Lab Anal; Hemodial Int; Clin Nephrol; Am J Nephrol; Nephron; Hemodial Int; J Nephrol. Analisando os locais de estudo 14 artigos foram realizados na Turquia (53,8\%), 4 artigos desenvolvidos na China (15,38\%), 2 realizados nos Estados Unidos, Taiwan e Japão (7,69\%), respectivamente. Espanha e Polônia foram publicados um artigo respectivamente $(3,84 \%)$.

Segundo o estudo de Okyayl et al. (2013), buscaram determinar a relação N / L em pacientes com Doença Renal Crônica (DRC), uma vez que a razão entre a contagem de neutrófilos e a contagem de linfócitos N / L foi examinada como uma nova medida de inflamação e demonstrou ter valores prognósticos e preditivos, especialmente naqueles com inflamação sistêmica. Nesse estudo foi elaborado um delineamento baseado em um grupo de pacientes que se encontravam em DRC prédiálise, pacientes em hemodiálise, pacientes em diálise peritoneal. Sendo assim, foi observado que razão N / L, as 
concentrações de IL-6 e proteína criativa de alta sensibilidade (PCR) foram significativamente maiores em todos os grupos de DRC em comparação com os indivíduos saudáveis, porém em indivíduos em hemodiálise e diálise peritoneal foi absolutamente maior. Além disso, os pacientes com DRC com hipertensão tinham uma relação N / L mais alta e níveis IL-6 maiores em comparação com aqueles sem hipertensão. Ademais, foi evidenciado que o aumento de leucócitos e seu componente de neutrófilo foram preditores significativos de mortalidade geral e associado a doença cardiovascular nos pacientes com diálise peritoneal. Portanto, a relação N / L apresenta associações significativas com marcadores bem conhecidos de inflamação, que após a validação em outros estudos clínicos a mesma possa ser usada na prática clínica.

Além disso, no estudo de Yuan et al. (2019), buscou-se investigar a associação da proporção de neutrófilos para linfócitos (NLR) com a progressão do estágio final da doença renal (ESRD), doença cardiovascular (DCV) e mortalidade por todas as causas em pacientes chineses com estágios 1-4 DRC. Para isso, 938 pacientes com DRC estágios 1-4, com idade variando de 18 a 74 anos (idade média de 52,8 anos), foram recrutados em 39 centros clínicos da China. A análise de regressão de Cox foi usada para estimar a associação entre o NLR e os resultados, incluindo ESRD, eventos cardiovasculares e mortalidade por todas as causas. Os dados foram obtidos por questionários, medidas antropométricas e coleta de sangue e urina, sendo que o tempo de acompanhamento desses pacientes foi de 4,55 anos. A análise de regressão de Cox revelou uma associação de NLR e o risco de ESRD apenas em pacientes com DRC em estágio 4, ao passo que não foi observada nenhuma associação significativa entre o NLR anormal e o risco de DCV ou mortalidade por todas as causas em pacientes com DRC no geral. Os resultados sugerem que a NLR está associada ao risco de ESRD em pacientes chineses com DRC em estágio 4.

Ademais, no estudo de Yoshimoti Q Nakayama (2019), buscou-se determinar se a NLR está associada a desfechos renais em pacientes com DRC. Para isso foi realizado um estudo observacional prospectivo entre julho de 2009 a novembro de 2016. A amostra continha 350 pacientes com DRC estágios 1-4 e foram excluídos os pacientes com qualquer malignidade, infecções agudas ou crônicas, doença inflamatória crônica, em uso de imunossupressores no momento da inscrição, exacerbação aguda de DRC e taxa de filtração glomerular estimada eTFG <15. Os pacientes foram divididos em dois grupos: NLR alto $(1,87$ a 5,92) e NRL baixo $(0,51$ a 1,86). Foi observado que o grupo com NLR alto teve maior risco de eventos adversos, visto que a relação neutrófilos/linfócitos está relacionada com a inflamação crônica e é um fator de risco independente para desfechos adversos. Além disso, a NLR relaciona-se com a PCR (elevada), TFGe (diminuída) e presença de Doença isquêmica do coração. Por seguinte, o estudo mostra que a NLR é um marcador útil para avaliar a progressão da doença renal e para prever a mortalidade e eventos cardiovasculares. Ademais na DRC, a inflamação crônica com níveis aumentados de CRP, IL- 6 e TNF- $\alpha$, bem como estresse oxidativo, foi associada a resultados renais adversos. Em conclusão, o estudo identificou o nível de NLR como um fator de risco independente para a progressão da doença renal em pacientes com DRC nos estágios 1-4. Assim, a medição do NLR pode ser útil para prever a progressão da doença renal em pacientes com DRC.

Em estudo conduzido por Altoneuren et. al (2018), investigaram se a razão neutrófilos/ linfócitos (NLR) está associado à progressão da DRC e a ascensão da doença à um estágio terminal, já que o marcador tem sido utilizado com preditor de morbimortalidade em outras doenças crônicas, assim como pode ocorrer na doença renal crônica (DRC). Para isso, foi realizado um estudo retrospectivo, a partir da análise de 740 pacientes com DRC entre os estágios 2 e 4 da doença. A partir disso, observou-se que o NRL médio dos pacientes analisados aumentou ao longo dos anos, assim como em consonância com o avanço do estágio da doença. Além disso, observou-se uma redução da estimativa da taxa de filtração glomerular (eTFG), maior proteinúria, menores níveis séricos de albumina e redução da sobrevida renal média, associada ao aumento do NRL. Entretanto, NLR não é um indicador independente para prever desfechos renais. Assim, conclui-se que o marcador é apenas uma medida bioquímica, que representa um nível mais elevado de inflamação na DRC avançada, indicando que a progressão pode ser mais rápida. Entretanto, outros fatores, com a TGF podem ser mais fidedignos para indicar progressão da DRC. 
Research, Society and Development, v. 10, n. 15, e508101523193, 2021

(CC BY 4.0) | ISSN 2525-3409 | DOI: http://dx.doi.org/10.33448/rsd-v10i15.23193

Quadro 1. Artigos levantados sobre relações celulares e doença renal crônica.

\begin{tabular}{|c|c|c|c|}
\hline PESQUISADORES & ANO & TIPO DE ESTUDO & OBJETIVOS DO ESTUDO \\
\hline KOÇYİĞìT et al & 2012 & Estudo observacional & $\begin{array}{c}\text { Avaliar a eficácia da relação neutrófilo/linfócitos (N / L) na previsão do ponto final da progressão para doença renal em estágio } \\
\text { terminal que requer terapia renal substitutiva (TRS). }\end{array}$ \\
\hline TURKMEN et al & 2012 & Estudo transversal & Determinar a relação entre NLR e inflamação em pacientes com ESRD \\
\hline TURKMEN E et al & 2013 & Estudo transversal & Determinar a relação entre PLR, NLR e inflamação em pacientes com ESRD. \\
\hline OKYAY et al. & 2013 & Estudo transversal & $\begin{array}{l}\text { O objetivo deste estudo foi investigar o valor da razão N / L como uma medida de inflamação sistêmica na população com DRC, } \\
\text { incluindo pacientes em pré-diálise, hemodiálise (HD) e diálise peritoneal (DP) em comparação com indivíduos saudáveis }\end{array}$ \\
\hline SOLAK et al. & 2013 & $\begin{array}{l}\text { Estudo coorte } \\
\text { observacional }\end{array}$ & $\begin{array}{l}\text { Avaliar se o NLR prediz a ocorrência de eventos cardiovasculares e se essa predição dependeria ou não de outros marcadores } \\
\text { inflamatórios como a PCR em pacientes com doenças renais crônicas em estágio 3-5. }\end{array}$ \\
\hline TURKMEN et al & 2014 & Estudo transversal & $\begin{array}{c}\text { Avaliar a relação entre calcificação da aorta torácica (TAC), calcificação da artéria coronária (CAC) e razão neutrófilos / } \\
\text { linfócitos (NLR) nesta população. }\end{array}$ \\
\hline BINNETOGLU et al & 2014 & Estudo caso-controle & Avaliar a relação entre proteinúria e NLR em pacientes com DRC sem DM. \\
\hline MALHOTRA et al & 2015 & Estudo transversal & $\begin{array}{l}\text { Avaliar se a NLR correlaciona com os níveis de PCR e determinar se uma combinação de marcadores inflamatórios (NLR e } \\
\text { níveis de albumina) melhora a previsão dos níveis de PCR nos pacientes com DRC (doença renal crônica) em hemodiálise. }\end{array}$ \\
\hline YAPRAK et al & 2016 & $\begin{array}{l}\text { Estudo observacional } \\
\text { prospectivo }\end{array}$ & Investigar a relação entre NLR, PLR e mortalidade por todas as causas em pacientes prevalentes em hemodiálise (HD). \\
\hline CHEN et al & 2016 & $\begin{array}{l}\text { Estudo observacional } \\
\text { prospectivo }\end{array}$ & Investigar os fatores prognósticos em pacientes com DRC avançada, incluindo DRT, com DAP em PTA em Taiwan \\
\hline $\begin{array}{l}\text { ERHAN TATAR e } \\
\text { CEM MIRILI }\end{array}$ & 2016 & $\begin{array}{l}\text { Estudo observacional } \\
\text { retrospectivo }\end{array}$ & $\begin{array}{c}\text { investigar a associação do rácio N/L e P/L com os resultados clínicos em pacientes geriátricos com fase 3-5 CKD que foram } \\
\text { acompanhados em clínicas ambulatoriais. }\end{array}$ \\
\hline SATO et al & 2017 & $\begin{array}{l}\text { Estudo observacional } \\
\text { retrospectivo }\end{array}$ & Avaliar a associação entre RNL e taxa de mortalidade em pacientes com nefropatia diabética em hemodiálise. \\
\hline LU et al & 2018 & $\begin{array}{l}\text { Estudo } \\
\text { transversal }\end{array}$ & $\begin{array}{c}\text { Avaliar a relação entre o NLR e os marcadores de rigidez arterial, incluindo cfPWV e AIx, e investigar a associação entre o } \\
\text { aumento do NLR e a mortalidade em pacientes em diálise peritoneal (DP). }\end{array}$ \\
\hline
\end{tabular}


Research, Society and Development, v. 10, n. 15, e508101523193, 2021

(CC BY 4.0) | ISSN 2525-3409 | DOI: http://dx.doi.org/10.33448/rsd-v10i15.23193

\begin{tabular}{|c|c|c|c|}
\hline ALTONEUREN et al & 2018 & $\begin{array}{l}\text { Estudo observacional } \\
\text { retrospectivo }\end{array}$ & Investigar NLR está associado à progressão da DRC a atingir uma doença renal em estágio terminal (ESRD). \\
\hline TONYALI et al & 2018 & $\begin{array}{l}\text { Estudo observacional } \\
\text { retrospectivo }\end{array}$ & Neste estudo, o objetivo foi investigar se NLR representa reserva renal e função após nefrectomia parcial ou radical. \\
\hline KUO et al & 2018 & Estudo transversal & $\begin{array}{c}\text { O objetivo deste estudo foi investigar se a NLR está associada à função renal e se essa associação muda com o envelhecimento } \\
\text { em uma população adulta de Taiwan. }\end{array}$ \\
\hline WOZIWODZKA et al & 2019 & $\begin{array}{l}\text { Estudo observacional } \\
\text { prospectivo }\end{array}$ & $\begin{array}{c}\text { Avaliar o impacto da proporção de neutrófilos para linfócitos (NLR) em longo prazo na mortalidade por todas as causas e } \\
\text { mortalidade cardiovascular (CV) em pacientes em hemodiálise (HD) }\end{array}$ \\
\hline YUAN et al & 2019 & $\begin{array}{l}\text { Estudo observacional } \\
\text { prospectivo }\end{array}$ & $\begin{array}{c}\text { Investigar a associação de NLR com a progressão do estágio final da doença renal (ESRD), doença cardiovascular (DCV) e } \\
\text { mortalidade por todas as causas em pacientes chineses com estágios 1-4 DRC }\end{array}$ \\
\hline $\begin{array}{l}\text { SEVENCAN; } \\
\text { OZKAN }\end{array}$ & 2019 & $\begin{array}{l}\text { Estudo observacional } \\
\text { prospectivo }\end{array}$ & $\begin{array}{c}\text { Investigar as associaçães entre a proporção de neutrófilos para linfócitos (NLR), a relação de plaquetas para linfócitos (PLR) e } \\
\text { taxa de filtração glomerular estimada (eTFG) em pacientes hipertensos com doença renal crônica (DRC) que não requerem } \\
\text { terapia de substituição renal. }\end{array}$ \\
\hline ZHANG et al & 2019 & Coorte & Investigar se a MLR elevada pode prever o risco do início do desenvolvimento da Doença Renal Crônica. \\
\hline $\begin{array}{l}\text { YOSHIOTI e } \\
\text { NAKAYAMA }\end{array}$ & 2019 & $\begin{array}{l}\text { Estudo observacional } \\
\text { prospectivo }\end{array}$ & Determinar se a NLR está associada a desfechos renais em pacientes com DRC. \\
\hline CATABAY E et al & 2020 & Estudo observacional & $\begin{array}{c}\text { Confirmar a associação de elevados valores de NLR e PLR à um estado inflamatório maior e predizer mortalidade entre } \\
\text { pacientes em hemodiálise. }\end{array}$ \\
\hline LI E et al & 2020 & Estudo transversal & $\begin{array}{c}\text { Avaliar a relação entre neutrófilos / linfócitos e plaquetas / linfócitos em associação à inflamação na doença renal em estágio } \\
\text { terminal (ESRD) sem diálise. }\end{array}$ \\
\hline VALGA et al. & 2020 & $\begin{array}{l}\text { Estudo transversal } \\
\text { multicêntrico }\end{array}$ & Avaliar a relação entre esses índices e a resistência à eritropoetina sobre a população em hemodiálise crônica. \\
\hline YILMAZ et al. & 2020 & Estudo transversal & Investigar NLR e MPV e sua relação com inflamação e proteinúria em pacientes com DRC \\
\hline CHEN e YANG & 2020 & $\begin{array}{l}\text { Estudo observacional } \\
\text { prospectivo }\end{array}$ & Investigar a relação entre o PLR e os eventos CVD em pacientes em diálise peritoneal ambulatorial contínua (CAPD) \\
\hline
\end{tabular}

Fonte: Autores (2021). 
Ademais, segundo o estudo Kocyigit et al. (2013), o objetivo foi avaliar a eficácia da relação neutrófilo/linfócitos (N / L) na previsão do ponto final da progressão para doença renal em estágio terminal que requer terapia renal substitutiva (TRS). Para isso, foi realizado um estudo observacional com 105 pacientes com DRC em estágio 4, os quais foram divididos em 2 grupos: grupo de proporção N / L baixa $(<3)$ e grupo de proporção $\mathrm{N} / \mathrm{L}$ alta $(>\geq 3,0)$. Além disso, teve como critérios de exclusão o início da diálise não planejada, doenças inflamatórias sistêmicas, leucopenia, uso de agente imunossupressor, doença hematológica, doenças malignas e doença autoimune. Assim, foi observado que pacientes com uma razão N / L alta $(>3,0)$ tiveram um pior prognóstico e progressão acelerada para a diálise, o aumento da contagem de neutrófilos e a redução da contagem de linfócitos está associado ao aumento da mortalidade em pacientes em hemodiálise e a relação $\mathrm{N} / \mathrm{L}$ foi considerada um marcador independente da taxa de progressão de pacientes com doença renal crônica estágio 4 para diálise. Portanto, a relação N / L é um importante biomarcador independente que correlaciona seu valor elevado com a rápida progressão da DRC para diálise e um excelente exame complementar para acompanhar a evolução dos pacientes com DRC para hemodiálise.

Além disso, o estudo conduzido por Kuo et al. (2018), buscou-se investigar se a proporção de neutrófilos para linfócitos (NLR) está associada à função renal e se essa associação muda com o envelhecimento em uma população adulta. Para isso, realizou-se um estudo transversal com uma amostra populacional de 2954 pessoas que compareceram a um exame de saúde em um centro médico no centro de Taiwan. Foram formados 4 grupos de acordo com sexo e idade ( $<60$ anos e $>60$ anos), sendo que comparações entre os grupos foram realizadas por meio do qui-quadrado ou teste de Fisher para variáveis categóricas e teste $t$ não pareado ou teste $U$ de Mann-Whitney para variáveis contínuas. Em comparação com participantes com idade $<60$, participantes com idade $\geq 60$ anos tiveram uma taxa de prevalência marcadamente maior de DRC em homens (7,6\% vs. $37,8 \%, p<0,001)$ e mulheres ( $3,8 \%$ vs. $28,0 \%$, p <0,001). Em homens com idade <60 anos a análise de regressão logística multivariada revelou que, após o ajuste para fatores de risco convencionais de DRC, maior NLR (por incremento de 1 unidade) foi independentemente associada com maior risco de DRC [OR ajustado = 1,48 (IC 95\%: 1,1 para 1,99, p = 0,009)] Essa mesma associação não ocorreu em homens e mulheres com $>60$ anos nem em mulheres com $<60$ anos. $\mathrm{O}$ estudo mostrou um efeito diferencial do envelhecimento na relação entre NLR e DRC em homens, mas não em mulheres. Por ser barato e prontamente disponível, o NLR pode ser potencialmente usado para avaliação de risco de DRC em homens com menos de 60 anos de idade.

Binnetoğlu et. al (2014), avaliou a relação entre a proteinúria e a razão neutrófilos/ linfócitos (NLR) em pacientes com doença renal crônica (DRC) sem diabetes mellitus (DM), uma vez que a proteinúria está muito associada a progressão da DRC. A relação NLR é, portanto, um marcador inflamatório muito importante para demonstrar essa progressão. Assim, o estudo realizado com diferentes grupos (I: pacientes com DRC sem proteinúria de 24h; II: pacientes com DRC e proteinúria; III: casos saudáveis), demonstrou que o NRL em pacientes do grupo 1 e 2 foi maior do que no grupo controle, sendo que a NRL afeta a proteinúria, independente de outros fatores de risco. Dessa maneira, concluiu-se que o a NRL é um marcador que representa valor prognóstico para existência e gravidade da proteinúria.

No estudo de Sato et al. (2017), propuseram avaliar a associação entre NRL e taxa de mortalidade em pacientes com nefropatia diabética em hemodiálise. Diante isso, foi realizado estudo observacional retrospectivo unicêntrico com 151 pacientes do sexo masculino de meia idade com nefropatia diabética. Esses foram divididos em dois grupos de acordo com os valores de NLR (NLR<3,5 e NLR>3,5) e foram coletados dados sobre a presença de infecção ativa, malignidade, hipertensão, peso e altura (mandatório para cálculo de IMC), doença cardíaca valvular grave, hemorragia gastrointestinal, paciente pósoperatório, hematoma e doença hematológica. Dessa forma, foi visto que pacientes com nefropatia diabética em diálise apresentam relação neutrófilo- linfócito elevada ditando menor sobrevida e evolução prognóstica pior.

Um estudo feito por Malhotra et al. (2015), buscaram avaliar se a NLR correlaciona com os níveis de PCR e 
determinar se uma combinação de marcadores inflamatórios (NLR e níveis de albumina) melhora a previsão dos níveis de PCR nos pacientes com DRC (doença renal crônica) em hemodiálise. Para isso, foi realizado um estudo transversal retrospectivo com 43.272 pacientes do sexo masculino dialisados de meia idade. Além disso, para análise, foi feito uma avaliação de dados pré-existentes no MONDO (Monitoramento de Resultados da Diálise). Assim, foi observado relação entre níveis elevados da NLR $(>5,0)$ com hipoalbuminemia $(<3,1)$, associação estatisticamente significativa entre os níveis de NLR e PCR $(>10,0)$ nos pacientes em diálise e o NLR ser melhor preditor inflamatório do que o PCR. Portanto, a NLR é um excelente biomarcador inflamatório em pacientes com DRC dialisados e apresenta benefícios prognósticos significativos, como seus valores elevados relacionando-se a redução plasmática de albumina.

No estudo Tukermen et al. (2012), buscaram determinar a relação entre NLR e inflamação em pacientes com ESRD. Para isso, foi realizado um estudo transversal com 61 pacientes (25 mulheres e 36 homens; idade média: 48,3 \pm 14,5 anos) recebendo diálise peritoneal (DP) ou hemodiálise (HD) por $\geq 6$ meses. Dessa forma, foram identificados níveis de NLR, PCR sérico, IL-6 e TNF- $\alpha$ foram significativamente maiores nos pacientes em DP quando comparados aos pacientes em HD. Pacientes com ESRD com NLR $\geq 3,5$ tiveram níveis de TNF- $\alpha$ significativamente maiores quando comparados com pacientes com NLR <3,5. Na análise de correlação bivariada, o NLR foi positivamente correlacionado com o TNF- $\alpha$ nesta população. Portanto, foi observado que o cálculo simples de NLR pode prever inflamação em pacientes com ESRD.

Outros estudos buscaram mostrar a correlação entre as razões inflamatórias e as comorbidades geradas pela DRC, como o estudo conduzido por Turkemen et al. (2014), teve o objetivo foi avaliar a relação entre calcificação da aorta torácica (TAC), calcificação da artéria coronária (CAC) e razão neutrófilos / linfócitos (NLR) nesta população. Diante disso, foi realizado um estudo transversal com 56 pacientes (22 mulheres, 34 homens; idade média, 49,9 \pm 14,2 anos) recebendo diálise peritoneal ou hemodiálise por $\geq 6$ meses. Houve uma correlação estatisticamente significativa entre NLR e escore calcificação da aorta torácica (TACS), contagens absolutas de neutrófilos e TACS em pacientes com ESRD. Em pacientes em HD, TACS está positivamente associado com NLR $(r=0,535, P=0,004)$, e escore calcificação da artéria coronária (CACS) também está positivamente correlacionado com NLR e idade avançada. Portanto, foi observado que o aumento da idade foi o único preditor independente de CACS de acordo com a análise de regressão linear e o cálculo simples de NLR pode prever calcificação vascular em pacientes com ESRD.

Além do mais, no estudo de Valga et al. (2020), a anemia é uma complicação bem frequente da DRC, dessa forma sabe-se que o índice de plaquetas-linfócitos (IPL) e neutrófilos-linfócitos (INL) são marcadores biológico de inflamação e disfunção endotelial. Baseando nesse quesito buscaram avaliar a relação entre os índices e a resistência a eritropoietina na população em hemodiálise crônica. Para isso, foi selecionado 397 indivíduos em hemodiálise crônica que apresentavam resistência a eritropoietina. Dessa forma, foi evidenciado que o aumento dos índices INL e IPL estavam associados a valores maiores de resistência à eritropoetina. Nesse contexto, conclui-se que os valores elevados dos índices INL e IPL foram associados a maiores valores de resistência a eritropoietina em uma população de alto risco cardiovascular.

Ademais, Woziwodzka et al. (2019), buscou-se avaliar o impacto da proporção de neutrófilos para linfócitos (NLR) em longo prazo na mortalidade por todas as causas e mortalidade cardiovascular (CV) em pacientes em hemodiálise (HD). Para isso, 84 pacientes com doença renal crônica (DRC) estágio 5, com mediana de idade de 61,5 (51,3-74,8) anos, foram incluídos no estudo e acompanhados por cinco anos, sendo estratificados em tercis de acordo com a NLR (mensurada a partir do hemograma completo) no início do estudo. Os pacientes com NLR $\geq 3,9$ (tercil mais alto) tiveram maior mortalidade por todas as causas em cinco anos do que os demais pacientes $(53,6 \%$ vs. 30,4\%; $\mathrm{p}=0,039)$. Por outro lado, apenas uma tendência para um aumento da mortalidade CV foi observada $(25,0 \%$ vs. $42,9 \%$; $=0,10)$. Depois de realizado ajuste para idade, sexo, diabetes (DM) e status de HD, NLR $\geq 3,9$ foi um preditor significativo de mortalidade por todas as causas em 5 anos (IC 95\%; $\mathrm{p}=0,017)$. O valor de corte ideal de NLR para predição de todas as causas de morte foi $\geq 4,16$ com sensibilidade e 
especificidade de 40,6\% e 86,5\%, respectivamente. Porém para o valor de corte usado de NLR $\geq 3,90$ a sensibilidade e a especificidade foram de $43,8 \%$ e $80,8 \%$, respectivamente. Não houve associação entre NLR e mortalidade devido à insuficiência cardíaca, infarto do miocárdio, infecções, neoplasias ou acidente vascular cerebral. Assim, concluiu-se que inflamação assintomática medida por NLR mostrou uma associação com mortalidade de longo prazo por todas as causas em pacientes com DRC estágio 5, mesmo enquanto a contagem de leucócitos estava ema faixa normal.

No estudo de Chen IC et. Al (2016), buscou-se investigar os fatores prognósticos em pacientes com DRC avançada, incluindo DRT, com doença arterial periférica (DAP) submetidos á angioplastia transluminal percutânea (PTA). Foi realizado um estudo retrospectivo e analisados $148 \mathrm{DRC}$ avançada ( $\mathrm{TFG} \leq 30$ ), incluindo $126 \mathrm{ESDR}$ em um total de 303 casos de TAP, no período de janeiro de 2011 a junho de 2014. O grau 6 de Rutherford, tanto NLR $<3,76$ ou NLR $\geq 3,76$, e uma história de hipertensão tiveram um impacto prognóstico positivo na ocorrência de mortalidade por todas as causas ou amputação maior. Por conseguinte, o nível de albumina mais alto $(\geq 3,0 \mathrm{mg} / \mathrm{dL})$ e sucesso técnico tiveram um efeito significativamente protetor em evitar a ocorrência de desfecho composto primário. Além disso, o histórico de hipertensão, seja NLR ou NLR maior ou igual a $\geq 3,76$, e a idade pode prever de forma significativa e independente a mortalidade. Em conclusão, NLR, mas não hsCRP ou PLR, pode ser um importante preditor de prognóstico de todos os principais desfechos clínicos em pacientes com DRC avançada e PAD recebendo PTA. Devido à sua disponibilidade conveniente, o NLR deve ser obtido e calculado em pacientes com DRC avançada com DAP. Novos estudos para estabelecer uma melhor estratégia e programa de saúde para prevenir ou modificar outros fatores merecem uma investigação mais aprofundada.

Outros estudos como Tonyalia et. al (2018), avaliaram se a razão neutrófilos/ linfócitos (NLR) poderia representar reserva e função renal após a realização de nefrectomia parcial ou radical, uma vez que a NRL é um marcador inflamatório envolvido na progressão da DRC. Assim, foi realizado um estudo retrospectivo com 240 pacientes, sendo estes divididos em grupos, tais como grupo de controles saudáveis (I), pacientes submetidos a nefrectomia parcial unilateral (II), pacientes que realizaram nefrectomina unilateral (III) e aqueles com doença renal crônica (DRC) (IV). Desta maneira, foi analisado que o valor médio da estimativa da taxa de filtração glomerular (eTFG) era mais baixa no grupo IV e maior no grupo II. O NRL e a creatinina se relacionaram à diminuição da reserva de néfron estimada, que diminui à medida que o número do grupo aumenta. Além disso, o valor de NRL > 3,18 foi associado a um risco 2,8 vezes maior desses pacientes apresentarem DRC. Conclui-se, portanto, que o NRL é um preditor de desenvolvimento de DRC em pacientes com nefrectomia parcial ou radical.

Lu et. al (2018), em seu estudo, avaliaram a relação entre razão neutrófilos/ linfócitos (NLR) e alguns marcadores de rigidez arterial, além de investigarem a associação entre o aumento do NRL em a mortalidade de pacientes com diálise peritoneal (DP), isso porque, a doença cardiovascular foi evidenciada como a principal complicação e causa de mortalidade em pacientes em diálise peritoneal. Assim, o estudo observacional transversal avaliou paciente com doença renal crônica em estágio terminal (ESRD) sem função residual do rim e tratamento com DP há pelo menos 3 meses. Observou-se que o NRL estava mais elevado pacientes que faleceram do que nos sobreviventes, que ele está associado a rigidez artéria na DP e que o sua elevação em pacientes com ESRD e na população em geral, é forte preditiva na gravidade e mortalidade da doença cardiovascular. Além disso, o NRL pode fornecer informações sobre rigidez arterial. Assim, o estudo concluiu que o NRL pode, de fato, ser um novo marcador de doença cardiovascular em pacientes com DP, apesar de que são necessárias mais pesquisas sobre $\mathrm{o}$ assunto.

No estudo de Solak et al. (2013), investigaram se a NLR prediz a ocorrência de eventos cardiovasculares em pacientes com DRC e se essa predição dependeria ou não de outros marcadores inflamatórios como a PCR, já que a relação é bastante segura na avaliação da ocorrência de eventos cardiovasculares adversos importantes em pacientes com síndromes coronárias agudas, insuficiência cardíaca e pacientes submetidos a procedimentos de revascularização coronária. À vista disso foi realizado um delineamento de 3 grupos de indivíduos, onde 70 pacientes estavam no estágio 3 da DRC, 74 no estágio 4 e 81 
pacientes no estágio 5. Foi evidenciado que NLR previu eventos cardiovasculares independentes da PCR, sugerindo papéis separados no processo inflamatório para esses dois biomarcadores. Além disso, a contagem de linfócitos mostrou uma redução significativa nos estágios da DRC do estágio 3 ao estágio 5, que foi associado ao aumento da inflamação. Concluiu-se então que que o NLR em pacientes com DRC, facilmente calculado a partir do sangue diferencial de rotina, fornece prognóstico (de diferentes aspectos do estado inflamatório geral) para o estado de inflamação de pacientes com DRC.

Por fim, além da razão neutrófilo-linfócito (NLR), há outros que também podem ser usados no prognóstico da DRC, como o apresentado no estudo de Chen e Yang (2020), o objetivo foi investigar a relação entre o PLR (Platelet-to-lymphocyte) e os eventos cardiovasculares em pacientes em diálise peritoneal ambulatorial contínua (CAPD). Para tal, foi realizada uma pesquisa, de fevereiro de 2014 a março de 2017, com 70 pacientes (29 mulheres), maiores de 18 anos, apresentando uremia e em CAPD regular a 3 meses. Os participantes com doenças hematológicas, doenças hepáticas, tumores malignos, doenças auto-imunes, disfunções da tireóide, e recentes infecções foram excluídos. A partir de então entendeu-se que o PRL elevado está relacionado com aumento da incidência de enventos cardiovasculares, independente dos fatores de risco e que a contagem de plaquetas dos pacientes com doenças cardiovasculares é maior quando comparados aos pacientes sem eventos cardiovasculares. Assim, a combinação de contagem reduzida de linfócitos e aumento de plaquetas pode ser usado como um novo indicador do estado inflamatório, fato importante pois a inflamação é um dos fatores que iniciam a fibrose tubularintersticial que pode levar a doença renal. Conclui-se que o PLR foi significativamente mais elevado no evento CVD do que o grupo de eventos não-CVD, indicando que o PLR estava intimamente relacionado a eventos CVD em pacientes com CAPD. Além disso, os eventos de PLR e CVD foram que se verificou estar independentemente correlacionada após o ajuste para fatores de risco, incluindo idade, hipertensão, diabetes, história de CVD, CRP e NLR.

Ademais, o estudo conduzido por Zhang et al. (2019), o objetivo foi investigar se a relação monócito/linfócito (MRL) elevada pode prever o risco do início do desenvolvimento da Doença Renal Crônica. Para isso, foi realizado um estudo coorte duplo cego com 1128 pacientes de meia idade do sexo masculino e feminino, os quais foram separados em grupos pelos tercis da relação M/L e N/L. Além disso, foram excluídos do estudo menores de 18 anos, pacientes sem lesão renal prévia, doenças cardiovasculares (infarto do miocárdio autorreferido e acidente vascular. Assim, foi possível relacionar o início recente da DRC com elevados parâmetros da relação M/L, pacientes nefropatas idosos e do sexo masculino apresentaram marcadores inflamatórios (leucócitos, linfócitos e monócitos) elevados e a relação M/L pode ser usada pela acompanhar a evolução do declínio da eTFG (estimativa de taxa de filtração glomerular). Portanto, o aumento da relação $\mathrm{M} / \mathrm{L}$ está intimamente relacionada ao risco de desenvolver DRC em pacientes com função renal prévia normal e seria de grande valia acrescentar esse parâmetro laboratorial para identificar precocemente a lesão renal.

Outros estudos buscaram avaliar mais de um marcador em conjunto, de modo a criar uma relação entre os mesmos, conforme o artigo feito por Yilmaz et al. (2017), buscaram mostrar que o volume plaquetário médio (VPM) e a razão neutrófilos / linfócitos (NLR) também aumenta durante a inflamação e pode estar associada a um pior prognóstico na DRC. Dessa forma, decidiram investigar a relação com a inflamação e proteinúria. Para isso, realizaram um delineamento com pacientes com DRC nos estágios 3 e 4 com idades entre 19 a 65 anos, comparando com indivíduos saudáveis. A partir disso, evidenciaram que a NLR é significativamente maior em pacientes com DRC em comparação com indivíduos saudáveis e o NLR aumenta com a progressão do estágio da doença renal e está relacionado com a proteinúria. Já em relação ao VPM não detectaram uma diferença estatisticamente significativa entre pacientes com DRC e pacientes controles saudáveis, mas observaram uma correlação negativa entre a taxa de filtração glomerular e VPM. Portanto, pode-se concluir que o NLR pode ser usado como uma indicação de inflamação e proteinúria na DRC.

Outro estudo realizado foi o de Sevencan e Ozkan (2019), e teve como intuito investigar as associações entre a proporção de neutrófilos para linfócitos (NLR), a relação de plaquetas para linfócitos (PLR) e taxa de filtração glomerular 
estimada (eTFG) em pacientes hipertensos com doença renal crônica (DRC) que não requerem terapia de substituição renal. Para isso, foi realizado um estudo prospectivo que incluiu 271 pacientes os quais foram divididos em 2 grupos: (a) aqueles com DRC estágios 1 e 2 e (b) aqueles com DRC estágio 3. Todos os pacientes foram avaliados quanto a presença de fatores de risco. Foi utilizado hemograma completo para mensurar o NLR e o PLR, além de serem medidos os níveis de albuminúria e ácido úrico. O NLR, albuminúria e ácido úrico foram maiores nos pacientes com DRC estágio 3 do que naqueles com estágio 1 e $2(\mathrm{p}=0,013, \mathrm{p}=0,001$ e $\mathrm{p}=0,001$, respectivamente), porém nenhuma diferença significativa foi detecdada na PLR. Em pacientes no estágio 3 , albuminúria e ácido úrico foram considerados fatores de risco independentes que afetam a eTFG $(\mathrm{p}=$ 0,042 e $p=0,001$, respectivamente). Os efeitos da NLR e PLR na eTFG não foram significativos ( $p=0,104$ e $p=0,578$, respectivamente). O NLR, semelhante à albuminúria e ao ácido úrico, mostrou-se um marcador específico para pacientes com DRC em estágio 3. No entanto, o NLR e PLR não atuaram como fatores de risco independentes que afetam o eTFG.

Além do mais, no estudo de Tatar e Mirili (2016), o propósito foi investigar a associação da relação neutrófilos / linfócitos e plaquetas / linfócitos com os desfechos clínicos em pacientes geriátricos com doença renal crônica em estágio 3-5 (DRC). Para isso foi realizado um estudo entre janeiro de 2008 e dezembro de 2010, no qual foram incluídos 165 pacientes com mais de 65 anos que acompanhados em ambulatório no estágio 3-5 da DRC (TFG <60). Os dados demográficos e laboratoriais dos pacientes eram obtidos de seus arquivos. Dados laboratoriais realizados em pelo menos três medições (basal, um ano e último controle valores de ambulatório) ao longo do estudo. A TFG foi calculada usando a fórmula MDRD. Os pacientes foram divididos em dois grupos com relação a relação N / L aumentada (grupo 1) e relação N / L estável (grupo 2) durante o acompanhamento. Os principais resultados do estudo demonstraram que a relação N/L é um fator independente para predição de mortalidade em pacientes geriátricos com doença renal e é um importante parâmetro para previsão da mortalidade e para avaliação do risco cardiovascular. Ademais, nesses pacientes há um aumento na relação P/L, porém não foi identificada qualquer correção independente para a mortalidade. Em conclusão, a relação N/L é um parâmetro importante em previsão de morte em doentes geriátricos com estágio 3-5 CKD uma vez que é simples, rentável e usado rotineiramente no campo da gerontologia e da nefrologia. Aumento da relação N/L pode dar pistas importantes sobre o mau prognóstico da doença durante o acompanhamento desses pacientes.

Outrossim, no estudo Yaprak et al. (2016), buscaram investigar a relação entre NLR, PLR e mortalidade por todas as causas em pacientes prevalentes em hemodiálise (HD). Para isso, foram selecionados 80 pacientes com DRC com idades de 18-90 anos e que foram submetidos a hemodiálise três vezes por semana durante pelo menos 3 meses. Os pacientes foram acompanhados prospectivamente por 24 meses. Dessa forma, foi observado que NLR foi positivamente correlacionado com PLR ( $r=0,532, p<0,001)$, proteína $\mathrm{C}$ reativa (CRP; $r=0,252, p=0,024)$, e idade $(r=0,252, p=0,024)$ e correlacionado negativamente com creatinina sérica $(r=-0,441, p<0,001)$. PLR foi correlacionado positivamente com PCR $(r=0,332, p=$ $0,003)$ e negativamente correlacionado com o nível de creatinina sérica $(\mathrm{r}=-0,238, \mathrm{p}=0,033)$. A mortalidade por todas as causas foi maior em pacientes com grupo de PLR alto em comparação com os pacientes com grupo de NLR baixo (18,8 vs. $7,5 \%, \mathrm{p}=0,031)$ e em pacientes com grupo de PLR maior em comparação com pacientes com grupo de PLR mais baixo (18,8 vs. $7,5 \%, \mathrm{p}=0,022)$. Portanto, embora tanto a NLR quanto a PLR estivessem associadas à mortalidade por todas as causas em pacientes prevalentes em HD, apenas a PLR poderia prever independentemente a mortalidade por todas as causas nessas populações.

Turkmen et al. (2013), também teve como objetivo determinar a relação entre PLR (a proporção de plaquetas / linfócitos), NLR (proporção de neutrófilos / linfócitos) e inflamação em pacientes com ESRD. Foi realizado um estudo transversal envolvendo 62 pacientes com ESRD recebendo diálise peritoneal (DP) ou hemodiálise (HD) por $\geq 6$ meses. Os níveis de PLR, NLR, proteína C reativa, TNF- $\alpha$ e IL-6 foram medidos. Os níveis de PLR, NLR, proteína C reativa de alta sensibilidade sérica, IL-6 e TNF- $\alpha$ foram significativamente maiores em pacientes com DP quando comparados com pacientes 
em HD. Pacientes com ESRD com PLR $\geq 140$ tiveram níveis significativamente maiores de NLR, IL-6 e TNF- $\alpha$ quando comparados a pacientes com PLR <139. Na análise de correlação bivariada, PLR foi positivamente correlacionado com NLR, IL-6 e TNF- $\alpha$ nesta população. Quando comparamos a associação de PLR e NLR com IL-6 ( $r=0,371, P=0,003$ vs. $r=0,263$, $\mathrm{P}=0,04$, respectivamente) e TNF- $\alpha(\mathrm{r}=0,334, \mathrm{P}=0,008$ vs. $\mathrm{r}=0,273, \mathrm{P}=0,032$, respectivamente), a PLR foi considerada superior à NLR em termos de inflamação em pacientes com ESRD. Portanto, o cálculo simples da PLR pode prever a inflamação melhor do que a NLR em pacientes com ESRD.

No estudo de $\mathrm{Li}$ et al. (2020), buscaram avaliar a relação entre a proporção de neutrófilos / linfócitos (NLR), proporção de plaquetas / linfócitos (PLR) e inflamação em pacientes com doença renal em estágio terminal (ESRD) em hemodiálise de manutenção (HD). Para isso, foi realizado estudo transversal com 100 pacientes com ESRD em HD de manutenção. Dados demográficos do paciente, peso seco, índice de massa corporal, duração da HD (meses), etiologia da ESRD, dose administrada de diálise (spKt / V), hemograma completo, bioquímica do sangue e marcadores inflamatórios incluindo hs-CRP (mg/L), TNF- $\alpha$ (pg/mL), NLR e PLR foram registrados em todos os pacientes e comparados em pacientes com níveis de PCR-us de $\leq 3 \mathrm{mg} / \mathrm{L}$ vs.> $3 \mathrm{mg} / \mathrm{L}$. Em comparação com pacientes com níveis mais baixos de hs-CRP, os pacientes com níveis de hs-CRP de> $3 \mathrm{mg} / \mathrm{L}$ apresentaram valores significativamente maiores para NLR (3,7 $\pm 0,2$ vs. 2,7 \pm $0,2, p<0,01)$ e PLR $(150,7 \pm 6,9$ vs. $111,8 \pm 7,0, p<0,001)$. Ambos NLR e PLR foram positivamente correlacionados com hsCRP ( $\mathrm{r}=0,333, \mathrm{p}=0,01 \mathrm{er}=0,262, \mathrm{p}=0,001$, respectivamente). Portanto, pacientes com ESRD em HD de manutenção possuem valores mais altos de NLR e PLR em pacientes com níveis mais elevados de inflamação, juntamente com uma correlação positiva significativa de NLR e PLR com os níveis de hs-CRP.

Em conclusão, o último estudo de Catabay et al. (2017), buscaram determinar que NLR (proporção de neutrófilos / linfócitos) e PLR (a proporção de plaquetas / linfócitos) são associados à inflamação e pode predizer mortalidade entre pacientes em hemodiálise. Para isso, foram selecionados 108,548 pacientes com ESRD e idade $\geq 18$ anos que iniciaram a diálise de $1^{\circ}$ de janeiro de 2007 a 31 de dezembro de 2011 e foi colhido amostras de sangue. O NLR foi associado a uma mortalidade mais elevada, que pareceu mais forte no modelo com variação no tempo vs. modelo basal. PLR exibiu uma associação em forma de $\mathrm{J}$ com mortalidade em ambos os modelos. NLR forneceu melhor previsão de mortalidade, além de dados demográficos, comorbidades e albumina sérica; $\triangle \mathrm{AUROC}$ e NRI para mortalidade em 1 ano (IC 95\%) foram 0,010 $(0,009-0,012)$ e $6,4 \%(5,5 \%-7,3 \%)$, respectivamente. Além disso, R2 ajustado (IC 95\%) para o modelo de Cox aumentou de $0,269(0,262-0,276)$ para $0,283(0,276-0,290)$ no modelo sem variação no tempo e de $0,467(0,461-0,472)$ para $0,505(0,500-$ 0,512) no modelo variável com o tempo. Houve pouco ou nenhum benefício em adicionar PLR para prever a mortalidade. Portanto, o NLR elevado em pacientes incidentes em hemodiálise previu mortalidade, principalmente no curto prazo. NLR, mas não PLR, forneceu benefício adicional na previsão de mortalidade junto com dados demográficos, comorbidades e albumina sérica e deve ser incluída nas abordagens de prognóstico.

\section{Considerações Finais}

Após a leitura e análise dos artigos selecionados, conclui-se que existem dados consistentes na literatura correlacionando as relações celulares RNL, RML, RPL com a Doença Renal Crônica. Devido ao baixo custo e sua disponibilidade conveniente, as relações celulares possuem boa aplicabilidade, visto que seus valores aumentados refletem inflamação sistêmica no portador de DRC, sendo esse um preditor morbimortalidade. Portanto, o custo benefício dessas relações, justificam seu uso na prática clínica após validação desses resultados em novos estudos clínicos. 


\section{Referências}

Aguiar, L. K., Prado, R. R., Gazzinelli, A., Malta, D. C. (2020) Fatores associados à doença renal crônica: inquérito epidemiológico da pesquisa nacional de saúde. Revista Brasileira de Epidemiologia, 23(1), 1-15, 2020. http://dx.doi.org/10.1590/1980-549720200044.

Altunoren O., Akkus G., Sezal D. T., Ciftcioglu M., Guzel F. B., Isiktas S., Torun G. I., Uyan M., Sokmen M. F., Sevim H. A., Sarısık F. N., Senel M. E., Erken E., Gungor O. (2018). Does neutrophyl to lymphocyte ratio really predict chronic kidney disease progression? Int Urol Nephrol. 51(1):129-137. 10.1007/s11255-018-1994-7.

Ammirati A. L. (2020). Chronic Kidney Disease. Revista da Associação Médica Brasileira, 66(1), 03-09, 2020. http://dx.doi.org/10.1590/1806-9282.66.s1.3.

Bastos M. G., Bregman R., Kirsztajn G. M. (2010). Doença renal crônica: frequente e grave, mas também prevenível e tratável. Rev. Assoc. Med. Bras. 2010, 56(2): 248-253. http://www.scielo.br/scielo.php?script=sci_arttext\&pid=S0104-42302010000200028\&lng=en.https://doi.org/10.1590/S010442302010000200028

Binnetoğlu E., Şengül E., Halhallı G., Dindar S., Şen H. (2014). Is neutrophil lymphocyte ratio an indicator for proteinuria in chronic kidney disease? J Clin Lab Anal. 28(6):487-92. 10.1002/jcla.21715.

Catabay C., Obi Y., Streja E., Soohoo M., Park C., Rhee C. M., Kovesdy C. P., Hamano T., Kalantar-Zadeh K. (2017). Lymphocyte Cell Ratios and Mortality among Incident Hemodialysis Patients. Am J Nephrol. 46(5):408-416. 10.1159/000484177.

Chen I. C., Yu C. C., Wu Y. H., Chao T. H. (2016). Elevated Neutrophil-to-Lymphocyte Ratio Predicts Intermediate-Term Outcomes in Patients Who Have Advanced Chronic Kidney Disease with Peripheral Artery Disease Receiving Percutaneous Transluminal Angioplasty. Acta Cardiol 32(5):532-41.

Chen T., Yang M. (2020). Platelet-to-lymphocyte ratio is associated with cardiovascular disease in continuous ambulatory peritoneal dialysis patients. Int Immunopharmacol. 78:106063.

De Sousa L. D., Lunardi Filho W. D., Lunardi V. L., Santos S. S., Dos Santos C. P. (2011). The nursing scientific production about the clinic: an integrative review. Rev Esc Enferm. USP. 45 (2): 494-500.

Galvão C. M., Sawada N. O., Trevizan M. A. (2004). Systematic review: a resource that allows the incorporation of evidence into nursing practice. Rev Latino-am Enfermagem. 17(4):758-764.

Kirsztajn G. M., Souza E, Romão Jr J. E., Bastos M. G., Meyer F., Andrada N. C. (2011). Doença Renal Crônica (Pré-terapia Renal Substitutiva): Diagnóstico. Projeto Diretrizes. Associação Médica Brasileira.

Kocyigit I., Eroglu E, Unal A, Sipahioglu MH, Tokgoz B, Oymak O, Utas C. (2013). Role of neutrophil/lymphocyte ratio in prediction of disease progression in patients with stage-4 chronic kidney disease. J Nephrol. 26(2):358-65. 10.5301/jn.5000152.

Kuo Y. T., Wang Y. Y., Lin S. Y., Chang W. D. (2018). Age and sex differences in the relationship between neutrophil-to-lymphocyte ratio and chronic kidney disease among an adult population in Taiwan. Clin Chim Acta. 486:98-103. 10.1016/j.cca.2018.07.025.

Lee, J. S., Kim, N. Y., Na, S. H., Youn, Y. H., \& Shin, C. S. (2018). Reference values of neutrophil-lymphocyte ratio, lymphocyte-monocyte ratio, plateletlymphocyte ratio, and mean platelet volume in healthy adults in South Korea. Medicine, 97(26), e11138. https://doi.org/10.1097/MD.0000000000011138

Li P., Xia C., Liu P., Peng Z., Huang H., Wu J., He Z. (2020). Neutrophil-to-lymphocyte ratio and platelet-to-lymphocyte ratio in evaluation of inflammation in non-dialysis patients with end-stage renal disease (ESRD). BMC Nephrol. 25,21(1):511. 10.1186/s12882-020-02174-0.

Lu X., Wang S., Zhang G., Xiong R., Li H. (2018). High Neutrophil-to-Lymphocyte Ratio is a Significant Predictor of Cardiovascular and All-Cause Mortality in Patients Undergoing Peritoneal Dialysis. Kidney Blood Press Res. 2018,43(2):490-499. 10.1159/000488696.

Malhotra R., Marcelli D., von Gersdorff G., Grassmann A., Schaller M., Bayh I., Scatizzi L., Etter M., Guinsburg A., Barth C., Marelli C., Carioni P., van der Sande F., Kooman J. P., Liu E., Toffelmire T., Wang Y., Thijssen S., Usvyat L. A., Kotanko P. (2019). Relationship of Neutrophil-to-Lymphocyte Ratio and Serum Albumin Levels with C-Reactive Protein in Hemodialysis Patients: Results from 2 International Cohort Studies. Nephron. 2015,130(4):263-70. $10.1159 / 000437005$.

Morsch C, Veronese F. J. V. (2011) Doença Renal Crônica: Definição e Complicações. Clinical \& Biomedical Research

Okyay G. U., Inal S., Oneç K., Er R. E., Paşaoğlu O., Paşaoğlu H., Derici U., Erten Y. (2012). Neutrophil to lymphocyte ratio in evaluation of inflammation in patients with chronic kidney disease. Ren Fail. 2013,35(1):29-36. 10.3109/0886022X.2012.734429.

Porto, J. R., Gomes, K. B., Fernandes, A. P. \& Domingueti, C. P. (2017). Avaliação da função renal na doença renal crônica. Revista Brasileira de Análises Clínicas 2017 outubro, 49(1). http://www.rbac.org.br/artigos/avaliacao-da-funcao-renal-na-doenca-renal-cronica/

Sato H., Takeuchi Y., Matsuda K., Kagaya S., Saito A., Fukami H., Ojima Y., Nagasawa T. (2017). Pre-Dialysis Neutrophil-Lymphocyte Ratio, a Novel and Strong Short-Term Predictor of All-Cause Mortality in Patients With Diabetic Nephropathy: Results From a Single-Center Study. Ther Apher Dial. 2017 Aug,21(4):370-377. 10.1111/1744-9987.12533.

Sevencan N. O., Ozkan A. E. (2019). Associations between neutrophil/lymphocyte ratio, platelet/lymphocyte ratio, albuminuria and uric acid and the estimated glomerular filtration rate in hypertensive patients with chronic kidney disease stages 1-3. Arch Med Sci. 15(5):1232-1239. 10.5114/aoms.2018.76262.

Solak Y., Yilmaz M. I., Sonmez A., Saglam M., Cakir E., Unal H. U., Gok M., Caglar K., Oguz Y., Yenicesu M., Karaman M., Ay S. A., Gaipov A., Turk S., Vural A., Carrero J. J. (2012). Neutrophil to lymphocyte ratio independently predicts cardiovascular events in patients with chronic kidney disease. Clin Exp Nephrol. 17(4):532-40. 10.1007/s10157-012-0728-x. 
Research, Society and Development, v. 10, n. 15, e508101523193, 2021

(CC BY 4.0) | ISSN 2525-3409 | DOI: http://dx.doi.org/10.33448/rsd-v10i15.23193

Tatar E., Mirili C., Isikyakar T., Yaprak M., Guvercin G., Ozay E, et al. (2016). The association of neutrophil/lymphocyte ratio and platelet/lymphocyte ratio with clinical outcomes in geriatric patients with stage 3-5 chronic kidney disease. Acta Clinica Belgica. 71(4):221-6.

Tonyali S., Ceylan C., Yahsi S., Karakan M. S. (2018). Does neutrophil to lymphocyte ratio demonstrate deterioration in renal function? Ren Fail. 40(1):209212. 10.1080/0886022X.2018.1455590.

Turkmen K., Guney I., Yerlikaya F. H., Tonbul H. Z. (2012). The relationship between neutrophil-to-lymphocyte ratio and inflammation in end-stage renal disease patients. Ren Fail. 34(2):155-9. 10.3109/0886022X.2011.641514.

Turkmen K., Ozcicek F., Ozcicek A., Akbas E. M., Erdur F. M., Tonbul H. Z. (2014). The relationship between neutrophil-to-lymphocyte ratio and vascular calcification in end-stage renal disease patients. Hemodial 18(1):47-53. 10.1111/hdi.12065.

Valga F., Monzón T., Henriquez F., Santana-Del-Pino A., Antón-Pérez G. (2019). Platelet-to-lymphocyte and neutrophil-to-lymphocyte ratios as markers of erythropoietin resistance in chronic haemodialysis patients: a multicentre cross-sectional study. Nefrologia (Engl Ed). 40(3):320-327. 10.1016/j.nefro.2019.09.007.

Yaprak M., Turan M. N., Dayanan R., Akın S., Değirmen E., Yıldırım M., Turgut F. (2016). Platelet-to-lymphocyte ratio predicts mortality better than neutrophil-to-lymphocyte ratio in hemodialysis patients. Int Urol Nephrol. 2016 Aug,48(8):1343-1348. 10.1007/s11255-016-1301-4.

Yilmaz G., Sevinc C., Ustundag S., Yavuz Y. C., Hacıbekiroglu T., Hatipoglu E., Baysal M. (2017).The relationship between mean platelet volume and neutrophil/lymphocyte ratio with inflammation and proteinuria in chronic kidney disease. Saudi J Kidney Dis Transpl. 2017 Jan-Feb,28(1):90-94. $10.4103 / 1319-2442.198152$.

Yoshitomi R., Nakayama M., Sakoh T., Fukui A., Katafuchi E., Seki M., et al. (2019). High neutrophil/lymphocyte ratio is associated with poor renal outcomes in Japanese patients with chronic kidney disease. Renal Failure. (1):238-43.

Yuan Q., Wang J., Peng Z., Zhou Q., Xiao X., Xie Y., Wang W., Huang L., Tang W., Sun D., Zhang L., Wang F., Zhao M. H., Tao L., He K., Xu H., CSTRIDE study group. (2019). Neutrophil-to-lymphocyte ratio and incident end-stage renal disease in Chinese patients with chronic kidney disease: results from the Chinese Cohort Study of Chronic Kidney Disease (C-STRIDE). J Transl Med. 2019 Mar 15,17(1):86. 10.1186/s12967-019-1808-4.

Whittemore R., Knafl K. (2005) The integrative review: updated methodology. J Adv Nurs, 52(5), 546-553, 2005.

Woziwodzka K., Dziewierz A., Pawica M., Panek A., Krzanowski M., Gołasa P., Latacz P., Burkat M., Kuźniewski M., Krzanowska K. (2019). Neutrophil-tolymphocyte ratio predicts long-term all-cause mortality in patients with chronic kidney disease stage 5. Folia Med Cracov. 59(4):55-70. 10.24425/fmc.2019.131380.

Zhang M., Wang K., Zheng H., Zhao X., Xie S., Liu C. (2019). Monocyte lymphocyte ratio predicts the new-onset of chronic kidney disease: A cohort study. Clin Chim Acta. 503:181-189. 10.1016/j.cca.2019.11.021. 